\title{
Assessing and stabilizing aberrant neuroplasticity in autism spectrum disorder: the potential role of transcranial magnetic stimulation
}

\author{
Pushpal Desarkar ${ }^{1,2 *}$, Tarek K. Rajji ${ }^{1,2}$, Stephanie H. Ameis ${ }^{1,2,3,4}$ and Zafiris Jeff Daskalakis ${ }^{1,2}$ \\ ${ }^{1}$ Department of Psychiatry, Centre for Addiction and Mental Health, University of Toronto, Toronto, ON, Canada, ${ }^{2}$ Temerty \\ Centre for Therapeutic Brain Intervention, Centre for Addiction and Mental Health, Toronto, ON, Canada, ${ }^{3}$ Department of \\ Psychiatry, The Hospital for Sick Children, University of Toronto, Toronto, ON, Canada, ${ }^{4}$ Research Imaging Centre, Campbell \\ Family Mental Health Research Institute, The Centre for Addiction and Mental Health (CAMH), Toronto, ON, Canada
}

\section{OPEN ACCESS}

Edited by:

Lena Palaniyappan,

University of Nottingham, UK

Reviewed by:

Kwang-Hyuk Lee,

University of Sheffield, UK

Lindsay M. Oberman,

Harvard Medical School, USA

Melissa Kirkovski,

Deakin University, Australia

${ }^{*}$ Correspondence:

Pushpal Desarkar,

Department of Psychiatry, Centre for

Addiction and Mental Health, University of Toronto, 1001 Queen Street West, Unit 4-4, Toronto, ON M6J 1H4, Canada pushpal.desarkar@camh.ca

Specialty section:

This article was submitted to

Neuropsychiatric Imaging and Stimulation, a section of the journal Frontiers in Psychiatry

Received: 09 July 2015

Accepted: 25 August 2015 Published: 09 September 2015

Citation: Desarkar P, Rajii TK, Ameis SH and Daskalakis ZJ (2015) Assessing and stabilizing aberrant neuroplasticity in

autism spectrum disorder: the potential role of transcranial magnetic

stimulation.

Front. Psychiatry 6:124. doi: 10.3389/fpsyt.2015.00124
Exciting developments have taken place in the neuroscience research in autism spectrum disorder (ASD), and results from these studies indicate that brain in ASD is associated with aberrant neuroplasticity. Transcranial magnetic stimulation (TMS) has rapidly evolved to become a widely used, safe, and non-invasive neuroscientific tool to investigate a variety of neurophysiological processes, including neuroplasticity. The diagnostic and therapeutic potential of TMS in ASD is beginning to be realized. In this article, we briefly reviewed evidence of aberrant neuroplasticity in ASD, suggested future directions in assessing neuroplasticity using repetitive TMS (rTMS), and discussed the potential of rTMS in rectifying aberrant neuroplasticity in ASD.

Keywords: autism spectrum disorder, transcranial magnetic stimulation, neuroplasticity, EEG, treatment

\section{Introduction}

Autism spectrum disorder (ASD) is a complex neurodevelopmental disorder characterized by persistent deficits in social communication and interaction and stereotyped behaviors, interests, and activities [Diagnostic and Statistical Manual of Mental Disorders, 5th Edition (DSM-5)] (1). The most recent US Centers for Disease Control and Prevention data estimate that ASD now affects 1 in 68 children (2). These data establish ASD as the most common neurodevelopmental disorder. Thus, the social, clinical, and economic burden of ASD is tremendous.

Since the turn of the century, significant advancements have been made in ASD research, and a range of macro- and micro-structural, neurochemical, functional, anatomic, and genetic abnormalities have been proposed [see reviews by Rubenstein and Merzenich (3), Parellada et al. (4), Chen et al. (5), Ameis and Catani (6)]; however, despite gaining important leads, the exact etiology of ASD is still unknown and successful treatment remains elusive. Thus, there is an urgent need to explore novel and effective investigational and mechanism-driven treatment paradigms for ASD.

One mechanism that has recently received a large amount of support suggesting its role in the pathophysiology of ASD is aberrant neuroplasticity $(7,8)$ In fact, several lines of evidence from genetic $(9-13)$ to animal model $(7,14)$, neuroimaging $(15,16)$, and brain stimulation $(17,18)$ research have all begun to implicate aberrant neuroplasticity in ASD. One neuroscientific tool that has become a widely used, safe, and non-invasive way to probe aberrant neuroplasticity is transcranial magnetic stimulation (TMS) and repetitive TMS (rTMS). Perhaps a fair example of this is the use of 
TMS/rTMS in the study of Parkinson's disease [see review by Shukla and Vaillancourt (19)], depression (20), and schizophrenia (21). The diagnostic and therapeutic potential of rTMS in ASD is beginning to be realized. In this article, we will briefly review evidence of aberrant neuroplasticity in ASD, suggest future directions in assessing neuroplasticity using rTMS, and discuss the potential of rTMS in rectifying aberrant neuroplasticity in ASD.

\section{Aberrant Neuroplasticity in ASD}

Before describing the evidence in favor of aberrant neuroplasticity in ASD, it may be worthwhile briefly revisiting neuroplasticity first. Neuroplasticity refers to neuron's ability to reorganize and alter their anatomical and functional connectivity in response to the environmental input. Long-term potentiation (LTP), which involves a net increase in synaptic efficacy, and long-term depression (LTD), which indicates a net decrease in synaptic efficacy, are the two prototypes of neuroplasticity (22).

In a simplistic model, LTP is mediated by glutamate via $N$-methyl-D-aspartate (NMDA) receptors (23). The basic process of LTP generation involves the removal of the $\mathrm{Mg}^{2+}$ block of the post-synaptic NMDA receptors by a strong wave of depolarization in the dendritic spine, leading to a rapid inflow of $\mathrm{Ca}^{2+}$ that activates several kinases, eventually leading to the generation of LTP. Similarly, LTD too perhaps is dependent on NMDA receptors. The mechanism of LTD generation, however, requires milder activation of post-synaptic NMDA receptors, which leads to an intermediate intracellular $\mathrm{Ca}^{2+}$ elevation (23). One key regulator of LTP and LTD is gamma-aminobutyric acid (GABA) released by the inhibitory interneurons (24). At the synaptic level, the fine balance between excitation (mediated by glutamate) and inhibition (mediated by GABA) could be crucial for optimal level of neuroplasticity (25).

\section{Evidence from the Structural Neuroimaging Studies in ASD}

Most of the symptoms of ASD develop in the first few years of life when synaptic development and maturation are occurring at a rapid rate, and one of the most consistent morphological findings that emerged from the structural neuroimaging studies in ASD is early brain overgrowth (15) [also see review by Courchesne et al. (16)]. Such atypical brain enlargement appears to be most pronounced between 2 and 5 years of age (16), and it preferentially affects the frontal and temporal cortices (5). Furthermore, recent evidence indicates that atypical cortical development in ASD subjects persists beyond toddlerhood. In particular, evidence of cortical thinning has been observed among adolescents and young adults (26). These observations led to the hypothesis that ASD is associated with a significant disruption of the typical synaptic maturation and plasticity (5).

\section{Evidence from the Genetic Studies in ASD}

Of all the proposed neurobiological theories of ASD, the potential contribution of genetic factors is backed by a large body of evidence [see review by Chen et al. (5)]. It is important to note that many ASD-associated genes reported by genome-wide association studies encode proteins related to synaptic formation, transmission, and neuroplasticity, and results from recent genetic studies involving ASD clients have consistently linked mutations involving several genes supporting synaptic maturation and neuroplasticity. The examples of such mutations involve genes critically involved in (a) synaptic maturation, e.g., neuroligin 3 and 4 (10), c3orf58, NHE9, and PCDH10 (13); (b) neuronal migration, e.g., CNTNAP2 (12); and (c) dendritic development, e.g., SHANK3 (12).

\section{Evidence from Animal Models of ASD}

Further evidence of aberrant neuroplasticity in ASD is shown by animal models. Perhaps one of the best known among these models is the valproic acid (VPA) rat model of autism. This model predicts that brain in ASD is likely to be hyperplastic. It has been found that, following a Hebbian Pairing Stimulation protocol, the amount of post-synaptic LTP measured in the neocortex and the amygdala doubled in VPA-treated rats compared with controls (14). However, other animal models utilizing genetically modified mice showed that ASD brain could be characterized by both impairment and enhancement of neuroplasticity. For example, Shank3(G/G) mice (27) and mice with MECP2 mutations (model of Rett's syndrome) (28) were shown to have cellular hypoplasticity, but mice with neuroligin-3 mutation were associated with hyperplasticity (29). Such divergent outcomes with regard to the direction of neuroplasticity in these animal experiments could be due to the nature of the genetic modifications used and their impact on the brain substrates of neuroplasticity. Nevertheless, a key insight emerging from these animal models is that if the brain becomes too much or too less plastic (i.e., hyper or hypo), cognition and behavior will be affected. It has been suggested that an optimum level of plasticity is necessary for optimal performance (30), and this process essentially involves keeping excitability within a normal physiological range (31).

\section{Excitation/Inhibition Imbalance in ASD}

Perhaps one of the widely cited neurobiological models in ASD over the past decade is the increased excitation/inhibition ratio in ASD brain (3). It has been suggested that the excitation-inhibition imbalance could be the key determinant of neuroplasticity abnormalities in neurodevelopmental disorders such as ASD (32), and a deficit in the inhibitory neurotransmission has been implicated in the etiopathogenesis of ASD [see review by Baroncelli et al. (25)]. It is believed that such deficits could develop during neuronal maturation (25). At the synaptic level, abnormally increased NMDA-mediated state of excitation, and/or abnormally reduced GABA-mediated inhibition, may lead to abnormally increased neuronal excitability and neuroplasticity. In fact, studies involving subjects with ASD have shown that excitatory glutamate receptors (NMDA and metabotropic glutamate receptor 5) are overexpressed, whereas inhibitory gamma aminobutyric acid $\mathrm{A}$ $\left(G_{A B A}\right)$ and $B\left(G_{A B A_{B}}\right)$ receptors are underexpressed in the $\operatorname{ASD}$ brain $(25,33)$. Additionally, post-mortem studies of minicolumnar morphometry in subjects with ASD also demonstrate a significant reduction of the peripheral neuropil space, which is the site of GABA-ergic lateral inhibition in the brain (34).

Transcranial magnetic stimulation has also been used to investigate excitation-inhibition imbalance in ASD. Specifically, 
paired-pulse TMS paradigms, involving the "pairing" of a "conditioning stimulus" with a "test stimulus" at different interstimulus intervals, have been used to assess cortical inhibition (CI) and facilitation. $\mathrm{CI}$ is the neurophysiological process in which inhibitory GABA-ergic interneurons selectively attenuate the activity of pyramidal neurons in the cortex. It has been suggested that $\mathrm{CI}$ is key to the regulation of neuroplasticity, and the therapeutic effects of rTMS could be mediated by the induction of local changes in $\mathrm{CI}$ (35). Emerging evidence indicates that post-synaptic $\mathrm{GABA}_{B}$ receptor-mediated $\mathrm{CI}$ is crucial for the regulation of neuroplasticity. $\mathrm{GABA}_{B}$ regulates neuroplasticity in two ways: (a) they contribute to the regulation of inhibition by mediating long-lasting inhibitory post-synaptic potentials (IPSPs) and (b) they reduce $\mathrm{GABA}_{\mathrm{A}}$ receptor-mediated inhibition through presynaptic autoinhibition of inhibitory interneurons (36). Using paired-pulse TMS paradigms, studies have found evidence for excitationinhibition imbalance in a subgroup of individuals with ASD (37, $38)$. Other studies have shown no abnormality in CI $(18,39)$ or a heterogeneous response to this paradigm (40). The heterogeneity in these findings reflects the known heterogeneity of ASD at both the behavioral and the physiological level.

\section{rTMS in the Assessment of Neuroplasticity in ASD}

Repetitive TMS, which involves repetitive delivery of pulses $(>1 \mathrm{~Hz})$, is used to modulate cortical activity for investigative and therapeutic purposes [see review by Kobayashi and Pascual-Leone (41)]. rTMS has been increasingly used to study neuroplasticity in humans. The basic premise is that rTMS can modulate activity in the targeted brain region for a duration that can outlast the effects of stimulation itself (30). It is believed that rTMS induces such lasting changes in the brain through altering neuroplasticity mechanisms (42). So far, two rTMS paradigms - theta-burst stimulation (TBS) (17) and paired associative stimulation (PAS) (18) - have been used to assess neuroplasticity in ASD.

\section{Theta-Burst Stimulation}

Theta-burst stimulation involves the delivery of a burst of three pulses at $50 \mathrm{~Hz}$ (i.e., $20 \mathrm{~ms}$ between stimulus) repeated at intervals of $200 \mathrm{~ms}$ (i.e., $5 \mathrm{~Hz}$, hence called theta-burst) (43). TBS comprises two well-established patterned stimulation protocols - continuous TBS (also known as cTBS) and intermittent TBS or iTBS. cTBS paradigm involves the delivery of continuous uninterrupted TBS for $40 \mathrm{~s}$. In the iTBS paradigm, a 2-s train of TBS is repeated every $10 \mathrm{~s}$ for a total of $190 \mathrm{~s}$. However, the total number of pulses delivered may vary from one study to another. In the original study, Huang et al. (43) used 600 pulses. iTBS produces sustained enhancement, whereas cTBS is associated with lasting suppression of cortical activity, indexed by potentiation and suppression of motor-evoked potential (MEP) following single-pulse TMS in the contralateral thumb muscle, respectively (43). It is believed that such lasting changes induced by iTBS and cTBS reflect LTP- and LTD-like mechanisms in the brain (43), and in previous experiments, they have been found to be mediated by NMDA receptor (44) and GABA receptor pathways (45), respectively.

\section{Paired Associative Stimulation}

Paired associative stimulation is another well-established rTMS paradigm that has been associated with the induction of LTP-like neuroplasticity (PAS-LTP). It has been shown that PAS-LTP is mediated by NMDA receptors (46). The PAS protocol involves the repetitive delivery of two paired (180 pairs at $0.1 \mathrm{~Hz}$ for $30 \mathrm{~min}$ ) stimulations: (1) an electrical peripheral nerve stimulation of the right median nerve, and $25 \mathrm{~ms}$ later, a (2) TMS pulse delivered to the contralateral motor cortex (M1) (hence PAS-25). PAS-25 results in LTP-like neuroplasticity that manifests as the potentiation of MEP in the thumb muscle following single-pulse TMS (46).

\section{Safety of rTMS in ASD}

Available limited data indicate that rTMS, when applied within established safety guidelines, is well tolerated and safe in both adult and pediatric ASD populations $(47,48)$. There is no current evidence of increased risk of seizure (48).

\section{rTMS Studies Assessing Neuroplasticity in ASD}

Asperger's disorder (AD), which was a subtype of the DSM-IV Pervasive Developmental Disorder, has now been subsumed under ASD in DSM-5 (1). A more direct evidence of aberrant neuroplasticity in $\mathrm{AD}$ subjects has been shown by recent rTMS studies using TBS and PAS paradigms. All these studies, however, have assessed neuroplasticity in the motor cortex (M1). One group found greater and long-lasting modulation of neuroplasticity (reflective of aberrant hyperplasticity) following both forms of TBS (cTBS and iTBS) in a small cohort (40) and, subsequently, in a relatively bigger sample of adults with AD (17). Another group, examining LTP-like neuroplasticity in a mixed cohort of adolescents and adults with AD using PAS, obtained similar results, i.e., aberrant neuroplasticity (18); however, the direction of aberrant neuroplasticity was different. In this study, it was found that, compared to typically developing subjects, PASinduced LTP-like plasticity was significantly deficient (reflective of aberrant hypoplasticity) in the $\mathrm{AD}$ group.

\section{Assessing Neuroplasticity in ASD Subjects Using rTMS: Future Considerations}

At present, research assessing neuroplasticity using rTMS in ASD population is at an early stage. Studies so far have only tested highfunctioning ASD subjects at the motor cortex (M1). Furthermore, findings obtained in the adult population may not be generalized to the pediatric population. For example, Oberman et al. (47) found a "paradoxical facilitatory effect" to cTBS in more than one-third of their sample consisting of children and adolescents. Therefore, to what extent current findings can be generalized is certainly not very clear at present. The potential factors that need to be considered by future research are heterogeneity in the ASD population, potential impact of the presence/absence of comorbidities including intellectual disabilities, medication use, developmental age, site of stimulation, stimulation parameters (e.g., TBS versus PAS), etc.

The other important point for consideration is that all existing studies utilizing rTMS have assessed neuroplasticity at the motor 
cortex (M1) of ASD brain. In the future, studies need to look at neuroplasticity in other potential areas of interest in the ASD brain. Information regarding which sites to choose for assessing neuroplasticity in ASD brain may come from existing rTMS intervention studies. So far, studies that used rTMS for therapeutic purposes to improve either symptoms or physiological and cognitive indices have focused on four areas of ASD brain - the dorsolateral prefrontal cortex (DLPFC), medial prefrontal cortex (mPFC), supplementary motor area, and right pars triangularis and pars opercularis [for a review see Oberman et al. (49)]. The DLPFC was chosen due to its extensive network connection with other specialized distributed and local networks in brain (34). Dorsomedial PFC ( $\mathrm{dmPFC})$ is another key area for stimulation since it is believed to be uniquely linked with the mentalizing ability (50). A recent trial of deep rTMS delivered bilaterally to the dmPFC significantly improved social relatedness in ASD subjects (51). Therefore, both DLPFC and mPFC could be potential sites of interest for studying neuroplasticity in ASD. Other brain areas related to mentalizing, such as the temporoparietal junction (TPJ) (52), and facial processing, such as superior temporal sulcus (53), could be potential sites for stimulation as well.

Establishing a stimulation paradigm to reliably assess neuroplasticity from these key areas of brain is challenging; however, the combination of TMS with electroencephalography (TMS-EEG) offers researchers an exciting opportunity to gather a more direct measure of neuroplasticity from these areas of brain. Previously, our group established that TMS-EEG can be a reliable method to measure neuroplasticity from $\mathrm{M} 1$ and also DLPFC (54). More recently, using a pioneering technique that combines PAS with EEG - "PAS-EEG," our group assessed and successfully demonstrated PAS-induced potentiation of cortical evoked activity, which is reflective of LTP-like neuroplasticity, in DLPFC (55). A similar TMS-EEG approach may be useful for studying neuroplasticity in other key areas of brain. For example, TBS can be combined with EEG to investigate neuroplasticity measures.

In the future, TMS-EEG can also be combined with various social-cognitive tasks and functional neuroimaging to better elucidate the brain-behavior relationship in ASD. Ultimately, TMSEEG will be combined with genetic research to better elucidate the link between underlying genetic factors (i.e., polymorphisms) and aberration in neuroplasticity captured more directly by TMS-EEG cortical readout. Results from a few early exploratory studies assessing the impact of single-nucleotide polymorphisms, e.g., brain-derived neurotrophic factor valine-to-methionine substitution at codon 66 (Val66Met) genotype (56), on TMSinduced plasticity measures have so far been encouraging.

\section{Can rTMS be Used as a Therapeutic Tool to Rectify Aberrant Neuroplasticity in ASD?}

Repetitive TMS affords researchers to design specific stimulation protocols that can modulate neuroplasticity, and such neuroplasticity-based brain stimulation interventions look promising. Recently, in a randomized double-blind sham-controlled study, our group demonstrated that application of 1,500 pulses/session of high-frequency $(20 \mathrm{~Hz})$ rTMS to DLPFC can "normalize" working memory deficits in schizophrenia (57). One possible mechanism of such improvement is enhancement of neuroplasticity in the DLPFC. There is a need to explore similar approach to treat aberrant neuroplasticity in ASD.

\section{What rTMS Stimulation Protocol to Choose for Stabilizing Aberrant Neuroplasticity in ASD?}

Since aberrant neuroplasticity has been linked with the pathogenesis of $\operatorname{ASD}(7,8)$, there is an urgent need to explore treatment paradigms that can stabilize aberrant neuroplasticity and thus potentially facilitate optimal social and cognitive performance and improve restricted and repetitive behaviors in ASD. In this regard, we would like to propose the potential role of extended dosing (i.e., 6,000 pulses) of high-frequency (i.e., $20 \mathrm{~Hz}$ ) rTMS (58).

In healthy adults, rTMS applied on M1 has been shown to enhance GABA-mediated inhibitory neurotransmission indexed by lengthening of the cortical silent period (CSP), a CI measure reflective of $\mathrm{GABA}_{\mathrm{B}}$-mediated inhibitory neurotransmission, with increased stimulation frequency. Our group found that the enhancement was maximal at $20 \mathrm{~Hz}$ (31). This finding breaks with convention that high-frequency stimulation results in excitation, whereas low-frequency stimulation results in inhibition, as $20-\mathrm{Hz}$ rTMS, but not $1-\mathrm{Hz}$ rTMS, resulted in a CSP prolongation $(31,58)$. One explanation is that $20-\mathrm{Hz}$ rTMS may exert its inhibitory effect by selectively affecting networks involving fastspiking inhibitory interneurons that mainly oscillate at higher (i.e., 30-70 Hz) frequencies (58). A recent study by our group investigating differing durations or doses of rTMS on CI in M1 in healthy subjects found that even a single session of extended dosing (6,000 pulses) with high-frequency $(20 \mathrm{~Hz})$ pulses led to significant lengthening of the $\mathrm{GABA}_{\mathrm{B}}$-mediated CSP compared with other paradigms (58). This effect was not seen with active or sham 1 - or $20-\mathrm{Hz}$ rTMS at 1,200 pulses or 3,600 pulses.

It has been suggested that, depending on the direction and magnitude of inhibition, $\mathrm{GABA}_{\mathrm{B}}$ receptor-mediated neurotransmission may attenuate neuroplasticity. In fact, baclofen, $a G_{B} A_{B}$ agonist, significantly attenuated LTP-like neuroplasticity in M1 induced by PAS (59). Since extended dosing (i.e., 6,000 pulses) of such specific high-frequency $(20 \mathrm{~Hz})$ rTMS protocol (58) appears to maximally enhance $\mathrm{GABA}_{\mathrm{B}}$-mediated inhibitory neurotransmission, one approach would be to assess if such protocols are able to stabilize aberrant hyperplasticity seen in ASD. This line of approach is also consistent with the excitation-inhibition imbalance in ASD, i.e., a general deficit in GABA-ergic inhibition, an increased excitation/inhibition ratio (3), and an evidence of reduced expression of $\mathrm{GABA}_{B}$ receptors (33). In the future, proofof-principle studies are needed to test this assumption. Because of its simplicity and reliability, such experiments may begin at M1 to see if the delivery of 6,000 pulses at $20 \mathrm{~Hz}$ can stabilize aberrant neuroplasticity in ASD subjects. If successful, further pilot studies will be required to assess whether rectifying aberrant neuroplasticity translates into actual clinical improvement or not. These pilot studies may potentially stimulate key areas of ASD brain discussed above, i.e., DLPFC, TPJ, and dmPFC, and determine key stimulation parameters, duration of sessions, etc. 


\section{Conclusion}

In summary, existing genetic and animal studies of ASD and evidence emerging from human rTMS studies have consistently indicated aberrant neuroplasticity in ASD brain. However, at this point, there are many unanswered questions regarding the exact etiopathological connection between aberrant neuroplasticity in the brain and development of autistic symptoms. Nevertheless, existing evidence still indicates that aberrant neuroplasticity could play a critical role in the pathogenesis of ASD. Therefore, it can be postulated that it may be possible to attain optimal social and cognitive performance in ASD by stabilizing aberrant neuroplasticity. In this context, we discussed a novel mechanism-driven approach toward achieving such goal using rTMS. If successful, this information will not only help us better understand the brain mechanisms involved in ASD but also stimulate trials testing mechanism-driven novel brain stimulation treatment paradigms for ASD.

\section{Acknowledgments}

PD is supported by the Innovation Fund from the Alternate Funding Plan of the Academic Health Sciences Centres of

\section{References}

1. American Psychiatric Association. Diagnostic and Statistical Manual of Mental Disorders 5th Edition (DSM-5). Washington, DC: American Psychiatric Association (2013).

2. Baio J. Prevalence of autism spectrum disorder among children aged 8 years-autism and developmental disabilities monitoring network, 11 sites, United States, 2010. MMWR Surveill Summ (2014) 63:1-21.

3. Rubenstein JL, Merzenich MM. Model of autism: increased ratio of excitation/ inhibition in key neural systems. Genes Brain Behav (2003) 2(5):255-67. doi:10.1034/j.1601-183X.2003.00037.x

4. Parellada M, Penzol MJ, Pina L, Moreno C, González-Vioque E, Zalsman G, et al. The neurobiology of autism spectrum disorders. Eur Psychiatry (2014) 29(1):11-9. doi:10.1016/j.eurpsy.2013.02.005

5. Chen JA, Penagarikano O, Belgard TG, Swarup V, Geschwind DH. The emerging picture of autism spectrum disorder: genetics and pathology. Annu Rev Pathol (2015) 10:111-44. doi:10.1146/annurev-pathol-012414-040405

6. Ameis SH, Catani M. Altered white matter connectivity as a neural substrate for social impairment in autism spectrum disorder. Cortex (2015) 62:158-81. doi:10.1016/j.cortex.2014.10.014

7. Markram K, Markram H. The intense world theory - a unifying theory of the neurobiology of autism. Front Hum Neurosci (2010) 21(4):224. doi:10.3389/ fnhum.2010.00224

8. Murdoch JD, State MW. Recent developments in the genetics of autism spectrum disorders. Curr Opin Genet Dev (2013) 23(3):310-5. doi:10.1016/j. gde.2013.02.003

9. Dolen G, Bear MF. Fragile X syndrome and autism: from disease model to therapeutic targets. J Neurodev Disord (2009) 1:133-40. doi:10.1007/ s11689-009-9015-x

10. Jamain S, Quach H, Betancur C, Råstam M, Colineaux C, Gillberg IC, et al. Mutations of the X-linked genes encoding neuroligins NLGN3 and NLGN4 are associated with autism. Nat Genet (2003) 34(1):27-9. doi:10.1038/ng1136

11. Tsai SJ. Is autism caused by early hyperactivity of brain derived neurotrophic factor? Med Hypotheses (2005) 65:79-82. doi:10.1016/j.mehy.2005.01.034

12. Durand CM, Betancur C, Boeckers TM, Bockmann J, Chaste P, Fauchereau $\mathrm{F}$, et al. Mutations in the gene encoding the synaptic scaffolding protein SHANK3 are associated with autism spectrum disorders. Nat Genet (2007) 39(1):90-8. doi:10.1038/ng1933
Ontario and Dean's Fund, Faculty of Medicine, University of Toronto. TR receives research support from Brain Canada, Brain and Behavior Research Foundation (previously known as NARSAD), Canadian Foundation for Innovation, Canadian Institutes of Health Research (CIHR), Ontario Ministry of Health and Long-Term Care, Ontario Ministry of Research and Innovation, the US National Institute of Health, and the Weston Brain Institute. SA receives financial support from the O'Brien Scholar's Program, an Ontario Mental Health Foundation New Investigator Fellowship, and The University of Toronto Dean's Fund New Staff Grant. In the last 5 years, ZD received research and equipment in-kind support for an investigator-initiated study through Brainsway Inc. ZD has also served on the advisory board for Hoffmann-La Roche Limited and Merck and received speaker support from Sepracor and Eli Lilly. This work was supported by the Ontario Mental Health Foundation (OMHF), the Canadian Institutes of Health Research (CIHR), the Brain and Behaviour Research Foundation, the Temerty Family and Grant Family, the Centre for Addiction and Mental Health (CAMH) Foundation, and the Campbell Institute. We would like to thank Dr. Amanda Sawyer for her contribution toward preparing this manuscript.

13. Morrow EM, Yoo SY, Flavell SW, Kim TK, Lin Y, Hill RS, et al. Identifying autism loci and genes by tracing recent shared ancestry. Science (2008) 321(5886):218-23. doi:10.1126/science.1157657

14. Markram K, Rinaldi T, LaMendola D, Sandi C, Markram H. Abnormal fear conditioning and amygdala processing in an animal model of autism. Neuropsychopharmacology (2008) 33:901-12. doi:10.1038/sj.npp.1301453

15. Courchesne E, Campbell K, Solso S. Brain growth across the life span in autism: age-specific changes in anatomical pathology. Brain Res (2011) 22(1380):138-45. doi:10.1016/j.brainres.2010.09.101

16. Courchesne E, Pierce K, Schumann CM, Redcay E, Buckwalter JA, Kennedy DP, et al. Mapping early brain development in autism. Neuron (2007) 56(2):399-413. doi:10.1016/j.neuron.2007.10.016

17. Oberman L, Eldaief M, Fecteau S, Ifert-Miller F, Tormos JM, Pascual-Leone A. Abnormal modulation of corticospinal excitability in adults with Asperger disorder. Eur J Neurosci (2012) 36:2782-8. doi:10.1111/j.1460-9568.2012.08172.x

18. Jung NH, Janzarik WG, Delvendahl I, Münchau A, Biscaldi M, Mainberger F, et al. Impaired induction of long-term potentiation-like plasticity in patients with high-functioning autism and Asperger syndrome. Dev Med Child Neurol (2013) 55(1):83-9. doi:10.1111/dmcn.12012

19. Shukla W, Vaillancourt DE. Treatment and physiology in Parkinson's disease and dystonia: using transcranial magnetic stimulation to uncover the mechanisms of action. Curr Neurol Neurosci Rep (2014) 14(6):449. doi:10.1007/ s11910-014-0449-5

20. Player MJ, Taylor JL, Weickert CS, Alonzo A, Sachdev P, Martin D, et al. Neuroplasticity in depressed individuals compared with healthy controls. Neuropsychopharmacology (2013) 38(11):2101-8. doi:10.1038/npp.2013.126

21. Rajji TK, Rogasch NC, Daskalakis ZJ, Fitzgerald PB. Neuroplasticity-based brain stimulation interventions in the study and treatment of schizophrenia: a review. Can J Psychiatry (2013) 58(2):93-8.

22. Bliss TVP, Cooke SF. Long-term potentiation and long-term depression: a clinical perspective. Clinics (Sao Paulo) (2011) 66(Suppl 1):3-17. doi:10.1590/ S1807-59322011001300002

23. Huerta PT, Volpe BT. Transcranial magnetic stimulation, synaptic plasticity and network oscillations. J Neuroeng Rehabil (2009) 6:1-10. doi:10.1186/1743-0003-6-7

24. Li R, Huang F, Abbas A, Wigstrom H. Role of NMDA receptor subtypes in different forms of NMDA-dependent synaptic plasticity. BMC Neurosci (2007) 8:55. doi:10.1186/1471-2202-8-55 
25. Baroncelli L, Braschi C, Spolidoro M, Begenisic T, Maffei L, Sale A. Brain plasticity and disease: a matter of inhibition. Neural Plast (2011) 2011:286073. doi:10.1155/2011/286073

26. Wallace GL, Eisenberg IW, Robustelli B, Dankner N, Kenworthy L, Giedd JN, et al. Longitudinal cortical development during adolescence and young adulthood in autism spectrum disorder: increased cortical thinning but comparable surface area changes. J Am Acad Child Adolesc Psychiatry. (2015) 54(6):464-9. doi:10.1016/j.jaac.2015.03.007

27. Speed HE, Kouser M, Xuan Z, Reimers JM, Ochoa CF, Gupta N, et al. Autismassociated insertion mutation (InsG) of Shank3 Exon 21 causes impaired synaptic transmission and behavioral deficits. J Neurosci (2015) 35(26):9648-65. doi:10.1523/JNEUROSCI.3125-14.2015

28. Moretti P, Levenson JM, Battaglia F, Atkinson R, Teague R, Antalffy B, et al. Learning and memory and synaptic plasticity are impaired in a mouse model of Rett syndrome. J Neurosci (2006) 26:319-27. doi:10.1523/ JNEUROSCI.2623-05.2006

29. Etherton M, Földy C, Sharma M, Tabuchi K, Liu X, Shamloo M, et al. Autismlinked neuroligin-3 R451C mutation differentially alters hippocampal and cortical synaptic function. Proc Natl Acad Sci U S A (2011) 108(33):13764-9. doi:10.1073/pnas.1111093108

30. Pascual-Leone A, Freitas C, Oberman L, Horvath JC, Halko M, Eldaief M, et al. Characterizing brain cortical plasticity and network dynamics across the age-span in health and disease with TMS-EEG and TMS-fMRI. Brain Topogr (2011) 24:302-15. doi:10.1007/s10548-011-0196-8

31. Daskalakis ZJ, Möller B, Christensen BK, Fitzgerald PB, Gunraj C, Chen R. The effects of repetitive transcranial magnetic stimulation on cortical inhibition in healthy human subjects. Exp Brain Res (2006) 174:403-12. doi:10.1007/ s00221-006-0472-0

32. Gatto CL, Broadie K. Genetic controls balancing excitatory and inhibitory synaptogenesis in neurodevelopmental disorder models. Front Synaptic Neurosci (2010) 2010(2):4. doi:10.3389/fnsyn.2010.00004

33. Fatemi SH, Folsom TD, Reutiman TJ, Thuras PD. Expression of GABA(B) receptors is altered in brains of subjects with autism. Cerebellum (2009) 8(1):64-9. doi:10.1007/s12311-008-0075-3

34. Casanova MF, Sokhadze E, Opris I, Wang Y, Li X. Autism spectrum disorders: linking neuropathological findings to treatment with transcranial magnetic stimulation. Acta Paediatr (2015) 104(4):346-55. doi:10.1111/apa.12943

35. Fitzgerald PB, Fountain S, Daskalakis ZJ. A comprehensive review of the effects of rTMS on motor cortical excitability and inhibition. Clin Neurophysiol (2006) 117(12):2584-96. doi:10.1016/j.clinph.2006.06.712

36. Deisz RA. The GABA(B) receptor antagonist CGP 55845A reduces presynaptic GABA(B) actions in neocortical neurons of the rat in vitro. Neuroscience (1999) 93:1241-9. doi:10.1016/S0306-4522(99)00203-1

37. Enticott PG, Rinehart NJ, Tonge BJ, Bradshaw JL, Fitzgerald PB. A preliminary transcranial magnetic stimulation study of cortical inhibition and excitability in high-functioning autism and Asperger disorder. Dev Med Child Neurol (2010) 52(8):e179-83. doi:10.1111/j.1469-8749.2010.03665.x

38. Enticott PG, Kennedy HA, Rinehart NJ, Tonge BJ, Bradshaw JL, Fitzgerald PB. GABAergic activity in autism spectrum disorders: an investigation of cortical inhibition via transcranial magnetic stimulation. Neuropharmacology (2013) 68:202-9. doi:10.1016/j.neuropharm.2012.06.017

39. Théoret H, Halligan E, Kobayashi M, Fregni F, Tager-Flusberg H, PascualLeone A. Impaired motor facilitation during action observation in individuals with autism spectrum disorder. Curr Biol (2005) 15(3):R84-5. doi:10.1016/j. cub.2005.01.022

40. Oberman L, Ifert-Miller F, Najib U, Bashir S, Woollacott I, Gonzalez-Heydrich J, et al. Transcranial magnetic stimulation provides means to assess cortical plasticity and excitability in humans with fragile $\mathrm{X}$ syndrome and autism spectrum disorder. Front Synaptic Neurosci (2010) 2:26. doi:10.3389/ fnsyn.2010.00026

41. Kobayashi M, Pascual-Leone A. Transcranial magnetic stimulation in neurology. Lancet Neurol (2003) 2:145-56. doi:10.1016/S1474-4422(03)00321-1

42. Ziemann U, Paulus W, Nitsche MA, Pascual-Leone A, Byblow WD, Berardelli A, et al. Consensus: motor cortex plasticity protocols. Brain Stimul (2008) 1:164-82. doi:10.1016/j.brs.2008.06.006

43. Huang YZ, Edwards MJ, Rounis E, Bhatia KP, Rothwell JC. Theta burst stimulation of the human motor cortex. Neuron (2005) 45:201-6. doi:10.1016/j. neuron.2004.12.033
44. Huang YZ, Chen RS, Rothwell JC, Wen HY. The after-effect of human theta burst stimulation is NMDA receptor dependent. Clin Neurophysiol (2007) 118:1028-32. doi:10.1016/j.clinph.2007.01.021

45. Stagg CJ, Wylezinska M, Matthews PM, Johansen-Berg H, Jezzard P, Rothwell JC, et al. Neurochemical effects of theta burst stimulation as assessed by magnetic resonance spectroscopy. J Neurophysiol (2009) 101(6):2872-7. doi:10.1152/jn.91060.2008

46. Stefan K, Kunesch E, Cohen LG, Benecke R, Classen J. Induction of plasticity in the human motor cortex by paired associative stimulation. Brain (2000) 123:572-84. doi:10.1093/brain/123.3.572

47. Oberman LM, Pascual-Leone A, Rotenberg A. Modulation of corticospinal excitability by transcranial magnetic stimulation in children and adolescents with autism spectrum disorder. Front Hum Neurosci (2014) 8:627. doi:10.3389/ fnhum.2014.00627. eCollection 2014.

48. Oberman LM, Enticott PG, Casanova MF, Rotenberg A, Pascual-Leone A, McCracken JT. Transcranial magnetic stimulation (TMS) therapy for autism: an international consensus conference held in conjunction with the international meeting for autism research on May 13th and 14th, 2014. Front Hum Neurosci (2015) 8:1034. doi:10.3389/fnhum.2014.01034. eCollection 2014.

49. Oberman LM, Rotenberg A, Pascual-Leone A. Use of transcranial magnetic stimulation in autism spectrum disorders. J Autism Dev Disord (2015) 45:524-36. doi:10.1007/s10803-013-1960-2

50. Gallagher HL, Frith CD. Functional imaging of 'theory of mind'. Trends Cogn Sci (2003) 7(2):77-83. doi:10.1016/S1364-6613(02)00025-6

51. Enticott PG, Fitzgibbon BM, Kennedy HA, Arnold SL, Elliot D, Peachey AA, et al. Double-blind, randomized trial of deep repetitive transcranial magnetic stimulation (rTMS) for autism spectrum disorder. Brain Stimul (2014) 7(2):206-11. doi:10.1016/j.brs.2013.10.004

52. Kennedy DP, Adolphs R. The social brain in psychiatric and neurological disorders. Trends Cogn Sci (2012) 16(11):559-72. doi:10.1016/j.tics.2012.09.006

53. Nomi JS, Uddin LQ. Face processing in autism spectrum disorders: from brain regions to brain networks. Neuropsychologia (2015) 71:201-16. doi:10.1016/j. neuropsychologia.2015.03.029

54. Daskalakis ZJ, Farzan F, Barr MS, Maller JJ, Chen R, Fitzgerald PB. Longinterval cortical inhibition from the dorsolateral prefrontal cortex: a TMSEEG study. Neuropsychopharmacology (2008) 33(12):2860-9. doi:10.1038/ npp. 2008.22

55. Rajji TK, Sun Y, Zomorrodi-Moghaddam R, Farzan F, Blumberger DM, Mulsant $\mathrm{BH}$, et al. PAS-induced potentiation of cortical evoked activity in the dorsolateral prefrontal cortex. Neuropsychopharmacology (2013) 38(12):2545-52. doi:10.1038/npp.2013.161

56. Cheeran B, Talelli P, Mori F, Koch G, Suppa A, Edwards M, et al. A common polymorphism in the brain-derived neurotrophic factor gene (BDNF) modulates human cortical plasticity and the response to rTMS. J Physiol (2008) 586:5717-25. doi:10.1113/jphysiol.2008.159905

57. Barr MS, Farzan F, Rajji TK, Voineskos AN, Blumberger DM, Arenovich T, et al. Can repetitive magnetic stimulation improve cognition in schizophrenia? Pilot data from a randomized controlled trial. Biol Psychiatry (2013) 73(6):510-7. doi:10.1016/j.biopsych.2012.08.020

58. de Jesus DR, Favalli GPD, Hoppenbrouwers SS, Barr MS, Chen R, Fitzgerald $\mathrm{PB}$, et al. Determining optimal rTMS parameters through changes in cortical inhibition. Clin Neurophysiol (2014) 125(4):755-62. doi:10.1016/j. clinph.2013.09.011

59. McDonnell MN, Orekhov Y, Ziemann U. Suppression of LTP-like plasticity in human motor cortex by the GABAB receptor agonist baclofen. Exp Brain Res (2007) 180:181-6. doi:10.1007/s00221-006-0849-0

Conflict of Interest Statement: The authors declare that the research was conducted in the absence of any commercial or financial relationships that could be construed as a potential conflict of interest.

Copyright (c) 2015 Desarkar, Rajji, Ameis and Daskalakis. This is an open-access article distributed under the terms of the Creative Commons Attribution License (CC $B Y)$. The use, distribution or reproduction in other forums is permitted, provided the original author(s) or licensor are credited and that the original publication in this journal is cited, in accordance with accepted academic practice. No use, distribution or reproduction is permitted which does not comply with these terms. 